\title{
El Taiji Quan y Qigong en la promoción de la salud y en el afrontamiento del estrés
}

\section{Taiji Quan and Qigong in promoting health and coping stress}

\author{
Jesús Carrillo ${ }^{1}$, Manuel Gómez-López ${ }^{2^{*}}$ y Gregorio Vicente ${ }^{3}$ \\ 1 Departamento de Investigación de SATSE-FUDEN en Murcia. \\ 2 Facultad de Ciencias del Deporte. Universidad de Murcia. Campus de Excelencia Internacional Regional "Campus Mare Nostrum". \\ 3 Facultad de Educación. Universidad de Murcia. Campus de Excelencia Internacional Regional "Campus Mare Nostrum".
}

\begin{abstract}
Resumen: El ejercicio y la actividad física mejoran la salud psicológica y física de los usuarios. Sin embargo, pocas veces se han examinado los efectos cuantitativos del Tai Chi Chuan en los sistemas cardiovascular, locomotor, mental, cognitivo y psicosocial entre otros. Se revisaron sistemáticamente los efectos sobre el estrés, la presión arterial y el riesgo cardiopulmonar, la capacidad respiratoria y su incidencia sobre patologías de este sistema, el equilibrio, la elasticidad muscular sobre el sistema locomotor, la ansiedad, la depresión y trastornos del estado de ánimo en las poblaciones orientales y occidentales. Se han considerado 53 estudios con una muestra de 5.875 sujetos y 13 meta análisis. Hay que resaltar que el Tai Chi parece estar asociado con mejoras en la gran mayoría de los sistemas que analizan los autores pero destaca el bienestar psicológico, incluyendo la reducción del estrés, ansiedad, depresión así como las mejoras a nivel musculo esquelético. Las conclusiones definitivas son limitadas debido a la variación en los diseños, los resultados diversos y los controles incorrectos de calidad. Son necesarios más ensayos aleatorios que garanticen la evidencia.

Palabras clave: Tai Chi, actividad física, estrés, bienestar psicológico.
\end{abstract}

Abstract: Exercise and physical activity improves psychological and physical health in users. However, rarely it is examined the quantitative effects of Tai Chi in cardiovascular, locomotor, mental, cognitive and psychosocial systems among others. The effects on stress, blood pressure and cardiopulmonary risk, breathing capacity and its impact on diseases of this system, balance, muscle elasticity on the locomotor system, anxiety, depression and disorders of systematically reviewed mood in both eastern and western populations. 53 studies were considered and 5875 subject were studied. Tai Chi appears to be associated with improvements in the vast majority of systems that analyze the authors but stresses the psychological well-being, including reducing stress, anxiety, depression and improvements to musculoskeletal level. Conclusion: these are limited due to variation in design, the various results and incorrect quality controls. Randomized trials are necessary to ensure the evidence.

Keywords: Tai Chi, physical activity, stress, psychological well-being.

\section{Antecedentes}

La promoción de la salud y el afrontamiento del estrés son dos conceptos que, aunque diferentes en sus términos, poseen una raíz común en su significado. El concepto de afrontamiento del estrés hace referencia a los esfuerzos conductuales y cognitivos que lleva a cabo la persona para afrontar al estrés; o lo que es lo mismo, la habilidad para manejar tanto las demandas externas como las internas generadoras del estrés, así como con el malestar psicológico que suele acompañar al mismo (Halsted, Bennet y Cunningham, 1993). Este concepto no puede entenderse como algo unitario, puesto que tiene diferentes acepciones en función de los investigadores. $\mathrm{El}$ afrontamiento es uno de los factores fundamentales implicados en el proceso del estrés que ha demostrado determinar significativamente sus consecuencias.

Relacionado con los estilos activos de afrontamiento destaca la actividad física que es un elemento esencial en las estrategias de afrontamiento del estrés y constituye una de las técnicas de afrontamiento más investigada y utilizada a nivel global. Con un afrontamiento activo basado en la actividad

Dirección para correspondencia [Correspodence address]: Manuel Gómez-López. Email: mgomezlop@um.es física se consigue, de forma categórica, un importante aumento de la salud percibida (Carrillo, 2013; Soto 2006). Son diversos los trabajos, que señalan los efectos positivos de la práctica de actividad física sobre la reducción del estrés, de la ansiedad y de los estados de depresión de leves a moderados que se verán en este artículo.

Diferentes investigaciones resaltan los efectos beneficiosos que la práctica de Taiji Quan y Qigong tienen sobre la salud (Chyu et al., 2010; Esch, Duckstein, Welke, Stefano y Braun, 2007; Johansson, Hassmén y Jouper, 2008), que con sus estudios han puesto de relieve los efectos observados que tienen sobre la salud. Se trata de un ejercicio físico tradicional que proviene de China, basado en una serie de movimientos armónicos unidos entre ellos con una secuencia continua en la que el cuerpo está constantemente cambiando de posición, con un centro de gravedad más bajo (Carrillo, Gómez-López y Vilchez, 2011). Durante su ejecución, se requiere una respiración profunda y una concentración mental para alcanzar la armonía entre el cuerpo y la mente. Es una modalidad de actividad física totalmente adaptable a personas con discapacidad y pacientes con enfermedades crónicas (Lan, Chen, Lai, y Wong, 2013). Por tanto, el objetivo del estudio es examinar la eficacia 
del Taichi Chuan y Qigong como técnicas de afrontamiento frente al estrés y comprobar su incidencia en aspectos físicos relacionados con la salud usando la revisión sistemática.

\section{Metodología}

\section{Estrategia de búsqueda}

Se efectuó una revisión sistemática con una búsqueda informatizada de la literatura médica en bases de datos en inglés: MEDLINE, PsycINFO, CAB, Health Star, Cochrane de Revisiones Sistemáticas, CINAHL, GlobalHealth y Alt HealthWatch; en español Cuiden, Cinhal, Latindex, Cuidatge, Catálogo CSIC; también se realizaron búsquedas las 3 principales bases de datos chinas, recomendadas por expertos en el dominio de la medicina basada en la evidencia en China, se incluyen: el Hospital de China Knowledge Database, China National Knowledge Infrastructure y Wanfang data desde enero de 2005 hasta febrero de 2017. También se realizaron búsquedas en las referencias de los artículos y comentarios seleccionados.

La búsqueda términos de esta revisión incluyó "Tai Chi”, "Tai Chi Chuan", "Tai Chi Chih", "tai chi”, "Tai Ji”, "Tai Ji Quan”, "Chi Kung”, "Qigong” y "taijiquan” (“Tai Chi” OR "Tai chi chuan” OR "tai chi” OR "Tai ji” OR "Tai ji quan” OR “Chi kung” OR "Qigong” OR “Taijiquan”) AND (“controlled trial” OR "random” OR ...). La selección de los estudios se realizó por artículos publicados que presentaron datos originales para estudios aleatorizados y ensayos controlados. La búsqueda arrojó un total de 145 artículos. Una vez eliminadas las duplicidades y considerando la ventana temporal fueron 53 los que se incluyeron y se observó, adicionalmente, 13 meta análisis y revisiones sistemáticas.

Se consideró como criterio de inclusión que las investigaciones fuesen investigaciones cuantitativas y que utilizaran el método del Tai Chi y/o del Qigong como terapia principal y que las fechas de publicación de los artículos estuviesen entre 2005 y 2017 y seguimiento superior a 4 semanas. Los criterios de exclusión fueron estudios cualitativos o no aleatorizados y con fechas de publicación anteriores a 2005 y seguimiento inferior a 4 semanas. El método de revisión fue realizado por dos revisores que de forma independiente seleccionaron los artículos. Se hizo una lectura crítica de la calidad metodológica basándose en los criterios de evaluación de la calidad de los estudios por el manual Cochrane (Higgins y Green, 2011).

\section{Desarrollo y Discusión}

Análisis de la promoción de la salud a través del Taiji Quan y Qigong

La diferencia fundamental entre los ejercicios de Taiji Quan y Qigong y aquellos realizados en Occidente radica en que los primeros no solo van dirigidos al sistema óseo y muscular, sino que también lo hacen a los órganos y sistemas internos. Tienen por tanto una concepción holística de la salud desde la medicina tradicional china y entienden que el beneficio sobre el componente físico, conlleva a su vez un beneficio sobre la mente y sobre el espíritu. Tanto una como otra práctica inciden en el control sobre la respiración como elemento esencial para poder trabajar los movimientos de forma lenta y suave, con un mínimo esfuerzo (Echevarría, 2008).

En relación con el estrés y con la práctica de Taiji Quan y Qigong, cualquier demanda, sea física, psicológica, externa o interna, buena o mala, provocaba una respuesta biológica del organismo estereotipada. Se observó que esta respuesta llevaba a cambios hormonales cuantificables, de forma que las modificaciones que estas secreciones hormonales eran las responsables de nuestras reacciones ante el estrés ya fuesen funcionales u orgánicas (Ho, Wan, Au-Yeung, Lo, Siu et al., 2014).

Análisis de los aspectos terapéuticos del Taiji Quan y Qigong

Con el fin de establecer un criterio de estudio de las investigaciones consultadas se ha visto la conveniencia de constituir una equivalencia entre Taiji Quan y Qigong en cuanto a que la práctica es similar. Es más, las investigaciones incorporan una mezcla de ambos sistemas a la hora de hacer los estudios. La combinación de ambas proporciona un mayor grado de evidencia indicando que estas dos actividades generan un amplio rango de beneficios para la salud (Jahnke, Larkey, Rogers y Lin, 2010, Carrillo 2013). Profundizando en este aspecto, algunas investigaciones examinadas arrojan que los efectos de Qigong sobre la salud parecen tener menor magnitud que los realizados usando la técnica del Taiji Quan (Cheung, Lo, Fong, Chan, Wong et al., 2005; Du, Dong, Zhang, Jin, Xu et al., 2015).. Sin embargo, la mayoría de los estudios utilizados en esta revisión demuestran resultados similares y no hay tendencias particulares que indiquen que uno tiene diferencias con el otro.

Se ha establecido una clasificación por los distintos sistemas corporales para facilitar la comprensión.

Incidencia sobre el sistema cardiovascular

Se afirmó por parte de los investigadores que la práctica regular de Taiji Quan y Qigong pudo retrasar el envejecimiento de la función cardiorrespiratoria. Ambas técnicas tienen un gran beneficio sobre la presión arterial y además resultaron eficaces para disminuir los factores de riesgo coronario, resultando por lo tanto adecuado para promover la salud de las personas mayores y personas con hipertensión arterial de etiología circulatoria o para mejorar la presión arterial desajustada (Cheung et al., 2005). 
Otras investigación sugirieron que resultaban de gran utilidad para la prevención de enfermedades cardiovasculares y, principalmente, que esta actividad física tenía potenciales beneficios para la rehabilitación cardíaca (Gao, 2013; Gong, 2009; Nery et al., 2014). Podía ser usado en aplicaciones clínicas alopáticas como complemento de diferentes patologías, como los desórdenes crónicos de corazón, problemas de las arterias coronarias y de la circulación en general (Cheng, 2006; Hui, 2009; Lee, M., Pittler, Taylor-Piliae y Ernst, 2007; Logghe, 2009; Sun, 2010).

Incidencia sobre el sistema pulmonar y respiratorio

La práctica habitual del Taiji Quan o Qigong mejoró la función pulmonar por diferentes mecanismos físicos de esta manera se favoreció la función de intercambio de oxígeno en los alveolos. En una respiración profunda el intercambio gaseoso aumenta y respirando menos veces, se permite que los músculos puedan relajarse lo suficiente, se fatiguen menos y ayuden a mantener la función respiratoria durante más tiempo (Carrillo 2013; Soto, 2006).

Según la Dra. Taylor-Piliae el Taiji Quan utiliza la respiración abdominal, porque el porcentaje de oxigenación obtenido por esta modalidad es mayor que en la torácica. Cuando el diafragma transversal estira un centímetro, cada inspiración aumenta en $250 \mathrm{ml}$. aproximadamente. La respiración torácica la producen los músculos dorsales, que causan fluctuaciones en el tórax y su consumo de oxígeno y calórico es mayor que en la respiración abdominal (Taylor-Piliae y Haskel, 2007).

Incidencia sobre el sistema locomotor

La incidencia sobre el sistema locomotor o capacidades físicomotrices ha sido estudiada por diferentes autores que concluyen que el uso Taiji Quan y Qigong proporciona un aumento de fuerza, resistencia y flexibilidad, siendo por tanto un sistema válido para adultos y personas mayores (Soto, 2006). Insisten en la importancia de mantener el nivel de estas capacidades, sobre todo en las extremidades inferiores ya que la disminución de fuerza y capacidad se considera un factor asociado al riesgo de caídas.

Normalmente, la práctica de esta modalidad no requiere instrumentos y utiliza solo el peso corporal como resistencia, además la práctica con las rodillas ligeramente flexionadas parece que implica una mayor demanda de fuerza que se equilibra con la baja velocidad de sus movimientos. Este lento movimiento y las posiciones bajas son las que determinan la mejora de la fuerza y la resistencia de las piernas (Soto 2006).

A tenor de los resultados, el Taiji Quan y el Qigong parecen ser un buen sistema para fortalecer el sistema muscular. Si a esta característica se le añade su lentitud y la no confrontación con objetos ni con personas, el resultado es un ejercicio seguro. Es más, puesto que sus practicantes no se lesionan durante la práctica, se podría afirmar que es un ejercicio válido para cualquier edad y así lo corroboraron los estudios realizados por Chwan-Li et al., 2007; Esch et al., 2007; Faber, Bosscher, Chin, Paw y Van Wieringen, 2006; Fransen, Nairn, Winstanley, Lam y Edmonds, 2007; Tsang y Hui-Chan, 2005; Wolf et al., 2006; Zhang, IshikawaTakata yamazaki, Morita y Ohta, 2006.

Otro aspecto que destacan los autores es el equilibrio en las personas que lo practican sobre todo si son personas mayores (más de 60 años) por las posibles caídas y sus consecuencias. La revisión bibliográfica realizada establece que existe relación directa entre la práctica de Taiji Quan y el equilibrio (11 de las investigaciones así lo recogen). La mejora del equilibrio está asociada a la propiocepción corporal, los mecanismos que intervienen en la estabilidad de la marcha, la respuesta neuromuscular de las piernas, el equilibrio estático, el equilibrio dinámico y la coordinación; en definitiva, el Taiji Quan puede ser prescrito como un medio adecuado para promocionar la salud en las personas mayores (Audette et al., 2006; Chwan-Li et al., 2007; Gatts y Woollacott, 2006; Johansson et al., 2008; Li, Fisher, Harmer y McAuley, 2005; Li et al., 2005; Liu, Miller, Burton y Brown, 2010; Liu, Li y Shnider, 2010; Maciaszek, Osiski, Szeklicki y Stemplewski, 2007; Tsang, Fung, Chan, Lee y Chan, 2006; Voukelatos, Cumming, Lord y Rissel, 2007; Zhang et al., 2006). Por otro lado, según Stenlund, Birgander, Lindahl, Nilsson y Ahlgren (2009), no debe dejarse de lado la mejora del movimiento de los brazos y el control de los mismos, además de la tonificación de hombros que progresa significativamente, sobre todo al caminar y al correr.

Por lo tanto, la faceta rehabilitadora de miembros y articulaciones en general es otro de los aspectos que tanto Qigong como del Taiji Quan mejora notablemente. Los ejercicios son lentos y exigen poco gasto de energía por lo que resulta adecuado para los estados de convalecencia de una enfermedad o incluso para su práctica durante la misma ya que su finalidad es mantener el tono muscular de las partes afectadas, si estas pueden moverse (Carrillo, Gómez-López y Vicente, 2009; Carrillo, Gómez-López y Vílchez,.2011). En el caso de enfermedades sistémicas que afecten a articulaciones como artritis reumatoide o esclerosis múltiple los estudios sostienen que los pacientes mejoran considerablemente (Lee, Pittler y Ernst, 2007; Song, Lee, Lam y Bae, 2010; Wang et al., 2005). Finalmente en las fibromialgias los beneficios reportados son evidentes según los autores (Hammond y Freeman, 2006; Hall, Maher, Latimer y Ferreira, 2009).

Incidencia sobre el sistema mental, cognitivo y psicosocial

Los resultados de diferentes estudios demuestran que la práctica habitual de Taiji Quan y Qigong aumenta considerable- 
mente la confianza y la fuerza de los músculos, por lo que reduce de manera significativa la posibilidad de caídas, pero lo más importante es la desaparición del miedo a caer reduciendo de manera notable otros tipos de conductas relacionadas con las fobias (McGibbon et al., 2005; Wang et al., 2009, 2010).

La vigorización de la autoestima ocasiona una importante motivación en los practicantes, lo que lleva al aumento de la concentración (Irwin et al., 2008; Lee et al., 2007, Liu y Yao, 2010; Nguyen y Kruse, 2012) y sobre todo relajación en los participantes. La estimulación del sistema parasimpático provocada por la respiración abdominal, mejora todo el sistema nervioso autónomo lo que hace aumentar los recursos, por mayor afluencia de oxígeno a las células de todo el cuerpo y a las del sistema nervioso, en especial. Esta circunstancia determina una mejora de las funciones psicológicas y psicosociales aumentando de esta manera la capacidad cognitiva y reduciendo la tensión arterial, la depresión (Cheung et al., 2005; Escalante, Saavedra, García-Hermoso, Silva y Barbosa, 2010; Irwin, Olmstead y Oxman, 2007), la ansiedad (Hammond y Freeman, 2006), el dolor de tipo neurológico, la demencia y algunos trastornos de la conducta. Además favorece la relación con los demás miembros del grupo de practicantes y favorece normalmente el buen humor por la sensación de euforia que provoca la relajación y el aumento de oxígeno circulante. Una vez desaparecido el síndrome del estrés, los recursos son más abundantes y por tanto el avance es sustancial (Taylor-Piliae et al., 2010; Taylor-Piliae y Haskell, 2007). Uno de los beneficios inmediatos de la realización de Qigong consiste en la serenidad y el bienestar que relatan los practicantes, entrando de una forma natural en un estado meditativo que determina que al finalizar las sesiones los alumnos abandonen los lugares de la práctica satisfechos y optimistas.

Finalmente resaltar que la realización de este tipo de ejercicios mejora los estados de nerviosismo propios de los insomnios y de los trastornos del sueńo, mejorando de esta manera la calidad de vida del practicante (Du, Dong, Zhang, Jin, Xu, et al., 2015; Irwin et al., 2008).

Incidencia sobre otros sistemas y patologías

Diferentes investigaciones demuestran mejoras sustanciales en pacientes con diferentes enfermedades como puede ser la diabetes. Se constató una mejoría de los parámetros que se midieron para controlar esta enfermedad como la hemoglobina glicosilada $A_{1} C$. El descenso de este parámetro en la gran mayoría de los pacientes fue superior a la del grupo control (Chwan-Li et al., 2007; Liu et al., 2010; Wang, 2008).

Igualmente, el Taiji Quan se reveló como una práctica adecuada para la rehabilitación de personas después de un ictus, apoplejía o hemorragia cerebral o cualquier otro daño cerebral (Blake y Batson, 2009; Gemmell y Leathem, 2006).
En el caso del sistema digestivo y excretor, se encontraron menos estudios que reportasen efectos mensurables en su funcionamiento, pero fue de destacar la erradicación de síntomas como náuseas y diarreas, ambas estudiadas en el caso de la enfermedad de Crohn y la patología asociada a determinados tumores. En relación con los tumores cancerígenos y su posterior rehabilitación, tanto el Qigong como el Taiji Quan aumentaron la función pulmonar, la capacidad de actividad diaria y la calidad de vida en pacientes después de la cirugía ya hubiese sido de pulmón o de mama (Mustian, Palesh y Flecksteiner, 2008; Peppone et al., 2010), así como de próstata. Así mismo, se progresó en las medidas de autopercepción de salud, de calidad de vida y de autoestima (Lee et al., 2007; Motivala, Sollers, Thayer y Irwin, 2006).

Del mismo modo se realizaron investigaciones con pacientes sometidos a diálisis, en las que se verificó incrementos objetivos en su calidad de vida (Mustata et al., 2005). Se observó la evidencia de que la práctica de Taiji Quan y sobre todo Qigong estabilizaba y hacía descender de forma significativa los niveles de lípidos y el colesterol, además de optimizar también el sistema inmunológico, tanto en personas inmuno-suprimidas como en las que habían sufrido otras patologías (Chang, Koo, Wu y Chen, 2017; Cheng, 2006; Irwin et al., 2007; Chang, Koo, Wu, Chen, 2017; Yang et al., 2008).

En aspectos más generales, se pudo establecer un incremento significativo de la calidad de vida de las personas que lo practicaban, siendo más notables cuanto peor son las condiciones de inicio de los practicantes (Song et al., 2010; TaylorPiliae y Haskell, 2007; Taylor-Piliae et al., 2010). Además, se constató un aumento significativo en la autopercepción física y mental, la autoevaluación positiva de la salud y la confianza en si mismo para la realización de las actividades de la vida cotidiana (Tsang y Hui-Chan, 2005; Wang et al., 2009).

Por otro lado, autores como Chyu et al. seńalan que el entrenamiento de resistencia y otros ejercicios con una carga añadida pueden aumentar la formación de hueso, motivo por el que estos ejercicios se han recomendado a mujeres posmenopáusicas tras observarse una mayor densidad ósea en aquellas que lo realizaban (Chyu et al., 2010). Los resultados de diferentes estudios señalaron que la pérdida de hueso se retrasó, reduciendo el número de fracturas de los practicantes de Taiji Quan en comparación con las que no lo hacían o hacían otra actividad física (Chan et al., 2004; Chen, Yeh y Lee, 2006; Shen et al., 2007; Song et al., 2010; Woo, Hong, Lau y Lynn, 2007)

Esta revisión ha identificado numerosos resultados con diferentes niveles de evidencia sobre la eficacia del Qigong y del Taiji Quan, incluida la salud ósea, estado cardiopulmonar, la función física, la prevención de caídas y el equilibrio, calidad de vida en general, la inmunidad y factores psicológicos tales como la ansiedad, la depresión y la autoestima. En definitiva, ambas actividades poseen unas características biomédicas 
beneficiosas para la mejora de la calidad de vida, es decir, un programa de Taiji Quan y de Qigong debe ser considerado como una estrategia adecuada de salud pública (Jahnke, Larkey y Rogers, 2010; Jahnke, Larkey, Rogers y Lin, 2010; Wolf et al., 2006).

\section{Tiempo de intervención con Taiji Quan y Qigong}

Una vez analizados los efectos que tuvo la práctica de estas modalidades de ejercicio físico, resultó necesario conocer el tiempo de intervención para que implicase una mejora sostenible en la salud. Lo complejo de ambas técnicas dificultó qué cronograma y con qué intensidad se podría haber utilizado. Básicamente, la dosis implica de forma esencial el tiempo de práctica, tiempo que a su vez depende de varios factores como es la duración de la intervención (horas que dura una sesión) y el número de veces que se repite a lo largo del tiempo. De los estudios analizados se obtuvo que intervenciones de 12 semanas de duración tienen marcados efectos en el equilibrio y la salud cardiovascular (McGibbon et al., 2005; Voukelatos, Cumming, Lord y Rissel, 2007), en cambio, aquellas a más a largo plazo se suelen utilizar en rehabilitación y prevención (Taylor-Piliae, Newell, Cherin, Lee, King et al., 2010).

Se estima que la mayor magnitud en los beneficios del Taiji Quan y Qigong aumenta con el tiempo ya que las habilidades de los practicantes mejoran con la práctica (Carrillo 2013, Du et al. 2015; Li, Fisher, Harmer y McAuley, 2005). Por tanto, a corto plazo los estudios prospectivos asiduamente pueden subestimar los beneficios potenciales del Taiji Quan y del Qigong (Soto, 2006). Ocasionalmente se recogen estudios donde se han controlado a largo plazo los resultados de las intervenciones y la definición de los estándares de investigación para este tipo de intervenciones no está aún clara, por lo que resulta poco concluyente en sus observaciones.

Las investigaciones revisadas indican que el periodo aproximado para realizar la forma básica de Taiji Quan se encuen- tra entre 8 y 12 semanas de práctica como media, aunque algunos lo sitúan entre 6 y 8 semanas (Carrillo, 2013, Soto 2006).

A nivel general, la estudios hablan de resultados específicos en 12 semanas de media, aunque mayores niveles del número de horas por semana estarían asociados a niveles de mejora aún mayores (Tsang y Hui-Chan, 2005). Por otro lado, la puesta en práctica de las sesiones oscila entre los 30, 60 y 90 minutos, incluyendo en estos tiempos tanto el calentamiento como la vuelta a la calma (Carrillo 2013; Du, Dong, Zhang, Jin, Xu et al., 2015). Finalmente, en otros estudios no se observaron mejoras significativas entre los grupos de tratamiento y el grupo de control debido a la corta duración de la intervención, de 6 a 8 semanas en vez de las 12 habituales o más, aunque en otros trabajos fueron especialmente significativos después de tan solo 8 semanas (Zhang et al., 2006).

En la Tabla 1 se pueden observar los estudios considerados en esta revisión y en la Tabla 2 los meta análisis y las revisiones sistemáticas consideradas.

\section{Conclusiones}

El Taiji Quan y el Qigong son métodos preventivos que de forma global u holística proporciona habilidades motoras y sensaciones propioceptivas globales que permiten que quien lo practica encuentre un mayor equilibrio físico y mental. El ejercicio físico, la reeducación en hábitos saludables, entre otros, conformarían parte de la riqueza de la terapéutica ante el estrés, aspectos que se incluyen en la práctica de estas técnicas en una sesión ordinaria. No obstante, los estudios son limitados debido a la variación en los diseńos, los resultados diversos y los controles incorrectos de calidad. Serían necesarios más ensayos aleatorios que garanticen un mayor grado de evidencia aunque cada vez se va incrementado la misma a tenor de lo observado en las revisiones sistemáticas.

No existen conflictos de intereses por parte de los autores.

Tabla 1. Resultados de la búsqueda realizada 53 artículos analizados.

\begin{tabular}{|c|c|c|c|c|c|c|}
\hline Autor & Titulo & Muestra & Edades & Intervención & Variables & Conclusiones \\
\hline $\begin{array}{l}\text { Audette, et } \\
\text { al. (2006). }\end{array}$ & $\begin{array}{l}\text { Tai chi versus brisk wal- } \\
\text { king in elderly women. }\end{array}$ & 19 & $\begin{array}{r}71.4 \\
+-4.5\end{array}$ & $\begin{array}{c}12 \text { semanas } \\
3 \text { por semana } \\
60 \text { minutos }\end{array}$ & $\begin{array}{l}\text { VO2, } \\
\text { SF36, } \\
\text { STAI... }\end{array}$ & $\begin{array}{l}\text { A short style of TCC was found to be an effec- } \\
\text { tive way to improve many fitness measures in } \\
\text { elderly women over a 3-month period. TCC } \\
\text { was also found to be significantly better than } \\
\text { brisk walking in enhancing certain measures } \\
\text { of fitness including lower extremity strength, } \\
\text { balance and flexibility. }\end{array}$ \\
\hline $\begin{array}{l}\text { Blake, H. y } \\
\text { Batson, M. } \\
\text { (2009). }\end{array}$ & $\begin{array}{l}\text { Exercise Intervention in } \\
\text { brain Injury: a pilot ran- } \\
\text { domized study of Tai Chi } \\
\text { Qigong. }\end{array}$ & 20 & todas & $\begin{array}{l}8 \text { semanas } \\
1 \text { por semana } \\
60 \text { minutos }\end{array}$ & $\begin{array}{l}\text { GHQ12 } \\
\text { movilidad, } \\
\text { percepción } \\
\text { humor... }\end{array}$ & $\begin{array}{l}\text { This study provides preliminary evidence that } \\
\text { a brief Qigong exercise intervention program- } \\
\text { me may improve mood and self-esteem for } \\
\text { individuals with traumatic brain injury. This } \\
\text { needs to be tested in a large-scale randomized } \\
\text { trial. }\end{array}$ \\
\hline
\end{tabular}




\begin{tabular}{|c|c|c|c|c|c|c|}
\hline Autor & Titulo & Muestra & Edades & Intervención & Variables & Conclusiones \\
\hline $\begin{array}{l}\text { Carrillo, J. } \\
\text { (2013). }\end{array}$ & $\begin{array}{l}\text { Promoción de la salud de } \\
\text { los empleados públicos de } \\
\text { la Región de Murcia: Pre- } \\
\text { vención del estrés a través } \\
\text { del Taiji Quan y Qigong. }\end{array}$ & 432 & $41+-10$ & $\begin{array}{l}52 \text { semanas } \\
2 \text { por semana } \\
120 \text { minutos }\end{array}$ & $\begin{array}{c}\text { GHQ12, } \\
\text { PSS14 }\end{array}$ & \\
\hline $\begin{array}{l}\text { Chan, K., } \\
\text { Qin, L., Lau, } \\
\text { M., Woo, J., } \\
\text { Au, S. et al. } \\
\text { (2004). }\end{array}$ & $\begin{array}{l}\text { A randomized, prospecti- } \\
\text { ve study of the effects of } \\
\text { tai chi chuan exercise on } \\
\text { bone mineral density in } \\
\text { postmenopausal women. }\end{array}$ & 132 & $\begin{array}{l}54+-3.5 \\
\text { mujeres }\end{array}$ & $\begin{array}{l}12 \text { meses } \\
5 \text { por semana } \\
45 \text { minutos }\end{array}$ & $\begin{array}{c}\text { Densidad } \\
\text { ósea }\end{array}$ & $\begin{array}{l}\text { This is the first prospective and randomized stu- } \\
\text { dy to show that a programmed TCC exercise } \\
\text { intervention is beneficial for retarding bone loss } \\
\text { in weight-bearing bones in early postmenopau- } \\
\text { sal women. Long-term follow-up is needed to } \\
\text { substantiate the role of TCC exercise in the pre- } \\
\text { vention of osteoporosis and its related fracture }\end{array}$ \\
\hline $\begin{array}{l}\text { Chang, Koo, } \\
\text { Wu y Chen } \\
(2017)\end{array}$ & $\begin{array}{l}\text { Effects of a } 12 \text {-week pro- } \\
\text { gram of Tai Chi exercise } \\
\text { on the kidney disease } \\
\text { quality of life and phy- }\end{array}$ & 20 & 25 & $\begin{array}{l}12 \text { semanas } \\
1 \text { por semana } \\
60 \text { minutos }\end{array}$ & $\begin{array}{l}\text { Calidad } \\
\text { de vida en } \\
\text { enfermos } \\
\text { de renales }\end{array}$ & $\begin{array}{l}\text { Improvements in the kidney disease quality of } \\
\text { life and physical functioning were observed in } \\
\text { Taiwanese patients on hemodialysis with a } 12 \text { - } \\
\text { week Tai Chi exercise intervention. }\end{array}$ \\
\hline
\end{tabular}
sical functioning of tients with end-stage renal disease on hemodialysis.

\begin{tabular}{|c|c|c|c|c|c|c|}
\hline $\begin{array}{l}\text { Chen, H.H. } \\
\text { Yeh, M.L. } \\
\text { y Lee, F.Y. } \\
(2006) \text {. }\end{array}$ & $\begin{array}{l}\text { The effects of Baduanjin } \\
\text { Qigong in the prevention } \\
\text { of bone loss for middle- } \\
\text { aged women. }\end{array}$ & 44 & $45+-5$ & 12 semanas & $\begin{array}{l}\text { Densidad } \\
\text { ósea }\end{array}$ & $\begin{array}{l}\text { this study demonstrates promising efficacy of } \\
\text { Ba duan jin in preventing bone loss commonly } \\
\text { occurring in middle-aged women. Thus, Ba- } \\
\text { duanjin is valuable for promoting and maintai- } \\
\text { ning the health status of middle-aged women }\end{array}$ \\
\hline $\begin{array}{l}\text { Cheung, } \\
\text { B.M., Lo, } \\
\text { J.L., Fong, } \\
\text { D.Y., Chan, } \\
\text { M.Y., Wong, } \\
\text { S.H. et al. }\end{array}$ & $\begin{array}{l}\text { Randomized controlled } \\
\text { trial of qigong in the } \\
\text { treatment of mild essen- } \\
\text { tial hypertension. }\end{array}$ & 88 & & 16 semanas & $\begin{array}{l}\text { Presión ar- } \\
\text { terial SF36, } \\
\text { ansiedad } \\
\text { STAI }\end{array}$ & $\begin{array}{l}\text { Guolin qigong and conventional exercise have } \\
\text { similar effects on blood pressure in patients } \\
\text { with mild hypertension. While no additional } \\
\text { benefits were identified, it is nevertheless an } \\
\text { alternative to conventional exercise in the non- } \\
\text { drug treatment of hypertension. }\end{array}$ \\
\hline
\end{tabular}
(2005).

\begin{tabular}{|c|c|c|c|c|c|c|}
\hline $\begin{array}{l}\text { Chwan-Li, } \\
\text { S., Du, F., } \\
\text { Esperat, M., } \\
\text { Irons, B., } \\
\text { Ming-C, C. } \\
\text { et al. (2007) }\end{array}$ & $\begin{array}{l}\text { Effect of Tai Chi exercise } \\
\text { on type } 2 \text { diabetes: a fea- } \\
\text { sibility study. }\end{array}$ & 25 & $\begin{array}{l}20-70 \\
\text { ańos }\end{array}$ & 12 semanas & $\begin{array}{l}\text { Hbalc, } \\
\text { sf36 auto- } \\
\text { eficacia }\end{array}$ & $\begin{array}{l}\text { Showed no significant effect of Tai Chi on } \\
\text { HbA1c and self-reported fasting blood glucose, } \\
\text { although there seemed to be a trend of lowered } \\
\text { HbAlc. Analysis of subjects' response sugges- } \\
\text { ted a positive experience for those who comple- } \\
\text { ted the intervention }\end{array}$ \\
\hline $\begin{array}{l}\text { Chyu, M.C., } \\
\text { James, C.R., } \\
\text { Sawyer, S.F., } \\
\text { Brismée, } \\
\text { J.M., Xu, } \\
\text { K.T. et al. } \\
(2010)\end{array}$ & $\begin{array}{l}\text { Effects of Tai Chi exerci- } \\
\text { se on posturography, gait, } \\
\text { physical function and } \\
\text { quality of life in postme- } \\
\text { nopausal women with os- } \\
\text { teopaenia: a randomized } \\
\text { clinical study. }\end{array}$ & 61 & 65 & $\begin{array}{l}24 \text { semanas } \\
3 \text { por semana } \\
60 \text { minutos }\end{array}$ & $\begin{array}{l}\text { Calidad } \\
\text { de vida, } \\
\text { sentadillas, } \\
\text { grado de } \\
\text { apertura } \\
\text { articular }\end{array}$ & $\begin{array}{l}\text { Tai chi exercise may reduce risk factors for falls } \\
\text { by increasing the stride width, and may im- } \\
\text { prove quality of life in terms of general health, } \\
\text { vitality and bodily pain in postmenopausal } \\
\text { women with osteopaenia. }\end{array}$ \\
\hline $\begin{array}{l}\text { Hosseini H } \\
\text { et al. (2011) }\end{array}$ & $\begin{array}{l}\text { The effect of Tai Chi exer- } \\
\text { cise on the sleep quality } \\
\text { of the elderly residents } \\
\text { in Isfahan, Sadeghieh el- } \\
\text { derly home. }\end{array}$ & 62 & $68.7-69$ & $\begin{array}{l}12 \text { semanas } \\
3 \text { por semana } \\
25 \text { minutos }\end{array}$ & $\begin{array}{l}\text { PSQI } \\
\text { score }\end{array}$ & $\begin{array}{l}\text { Consistent with other studies, Tai chi exercise } \\
\text { may have a positive, significant effect on sleep } \\
\text { quality of life in older people. }\end{array}$ \\
\hline $\begin{array}{l}\text { Irwin et al. } \\
(2008)\end{array}$ & $\begin{array}{l}\text { Improving sleep quality } \\
\text { in older adults with mo- } \\
\text { derate sleep complaints: } \\
\text { a randomized controlled } \\
\text { trial of Tai Chi. }\end{array}$ & 102 & 69.9 & $\begin{array}{l}16 \text { semanas } \\
3 \text { por semana } \\
40 \text { minutos }\end{array}$ & PSQI & $\begin{array}{l}\text { Tai Chi Chuan has a positive role in impro- } \\
\text { ving self-rated sleep quality among older adults } \\
\text { with moderate sleep complaints, with the po- } \\
\text { tential to ameliorate sleep complaints possibly } \\
\text { before syndrome insomnia develops. }\end{array}$ \\
\hline
\end{tabular}




\begin{tabular}{|c|c|c|c|}
\hline $\begin{array}{l}\text { Taylor-Piliae, } \\
\text { R.E. y } \\
\text { Haskell, W. } \\
(2007) \text {. } \\
\end{array}$ & $\begin{array}{l}\text { Tai Chi exercise and } \\
\text { stroke rehabilitation. }\end{array}$ & $\begin{array}{l}\text { Meta } \\
\text { análisis }\end{array}$ & \\
\hline $\begin{array}{l}\text { Wang, C., } \\
\text { Bannuru, R., } \\
\text { Ramel, J., } \\
\text { Kupelnick, } \\
\text { B., Scott, T. } \\
\text { y Schmid, } \\
\text { C.H. (2010). }\end{array}$ & $\begin{array}{l}\text { Tai Chi on psychological } \\
\text { well-being: systematic } \\
\text { review and meta-analysis. }\end{array}$ & $\begin{array}{l}\text { Meta } \\
\text { análisis }\end{array}$ & \\
\hline $\begin{array}{l}\text { Wang, F., } \\
\text { Lee, E.K., } \\
\text { Wu, T., } \\
\text { Benson, H., } \\
\text { Fricchione, } \\
\text { G. et al. } \\
\text { (2014). }\end{array}$ & $\begin{array}{l}\text { The effects of tai chi on } \\
\text { depression, anxiety, and } \\
\text { psychological well-being: } \\
\text { a systematic review and } \\
\text { meta-analysis. }\end{array}$ & $\begin{array}{l}\text { Meta } \\
\text { análisis }\end{array}$ & \\
\hline $\begin{array}{l}\text { Wang, W., } \\
\text { Zhang, A., } \\
\text { Rasmussen, } \\
\text { B., Lin, L., } \\
\text { Kang, S. et } \\
\text { al. (2009). }\end{array}$ & $\begin{array}{l}\text { The effect of Tai Chi on } \\
\text { psychosocial well-being: } \\
\text { a systematic review of } \\
\text { randomized controlled } \\
\text { trials. }\end{array}$ & $\begin{array}{l}\text { Meta } \\
\text { análisis } \\
13 \text { estu- } \\
\text { dios }\end{array}$ & $\begin{array}{l}\text { It is still premature to make any conclusive } \\
\text { remarks on the effect of Tai Chi on psychoso- } \\
\text { cial well-being. }\end{array}$ \\
\hline
\end{tabular}

\section{Referencias}

1. Audette, J. F., Jin y. S., Newcomer, R., Stein, L., Duncan, G. et al. (2006). Tai chi versus brisk walking in elderly women. Age y Ageing, 35(4), 388-93.

2. Blake, H. y Batson, M. (2009). Exercise Intervention in brain Injury: a pilot randomized study of Tai Chi Qigong. Clinical Rehabilitation, 23(7), 589-98

3. Carrillo, J., Gómez-López, M. y Vicente, G. (2009). Mejora de la calidad de vida de los mayores a través del Tai Chi y Chi Kung. Retos. Nuevas tendencias en Educación Física, Deporte y Recreación, 16, 86-91.

4. Carrillo, J., Gómez-López, M. y Vílchez, P. (2011). Propuesta práctica ante la discapacidad. El uso del Tai Chi y del Qigong. Enfermería Global, 10(1), 1-14.

5. Carrillo, J. (2013). Promoción de la salud de los empleados públicos de la Región de Murcia: Prevención del estrés a través del Taiji Quan y Qigong. Tesis doctoral. Universidad de Murcia. Murcia.

6. Chan, K., Qin, L., Lau, M., Woo, J., Au, S. et al. (2004). A randomized, prospective study of the effects of tai chi chun exercise on bone mineral density in postmenopausal women. Archives of Physical Medicine and Rehabilitation, 85(5), 717-22.

7. Chang, J.H., Koo, M., Wu, S.W. y Chen, C.Y. (2017). Effects of a 12week program of Tai Chi exercise on the kidney disease quality of life and physical functioning of patients with end-stage renal disease on hemodialysis. Complementary Therapeutic Medicine. (2)30:79-83.

8. Chen, H.H., Yeh, M.L. y Lee, F.Y. (2006). The effects of Baduanjin Qigong in the prevention of bone loss for middle-aged women. American Journal of Chinese Medicine, 34(5), 741-47.

9. Cheng, T. (2006). Tai Chi: the chinese ancient wisdom of an ideal exercise for cardiac patients. International Journal of Cardiology, 117(3), 293-95.

10. Cheung, B.M., Lo, J.L., Fong, D.Y., Chan, M.Y., Wong, S.H. et al. (2005). Randomised controlled trial of qigong in the treatment of mild essential hypertension. Journal of Human Hypertension, 19(9), 697-704.

11. Chwan-Li, S., Du, F., Esperat, M., Irons, B., Ming-C, C. et al. (2007). Effect of Tai Chi exercise on type 2 diabetes: a feasibility study. Integrative Medicine Insights, 2, 15-23.

12. Chyu, M.C., James, C.R., Sawyer, S.F., Brismée, J.M., Xu, K.T. et al. (2010). Effects of Tai Chi exercise on posturography, gait, physical function and quality of life in postmenopausal women with osteopaenia: a randomized clinical study. Clinical Rehabilitation, 24(12), 10801090 .

13. Du, S., Dong, J., Zhang, H., Jin, S., Xu, G. et al. (2015). Taichi exercise for self-rated sleep quality in older people: a systematic review and meta-analysis. International Journal of Nurses Studies. 52(1):368-79.

14. Echevarría, P. (2008). Análisis antropológico de los patrones de uso y el perfil del usuario de terapias complementarias orientales. Gazeta de Antropología, 24(2), 1-18.

15. Escalante, Y., Saavedra, J.M., García-Hermoso, A., Silva, A.J. y Barbosa, T.M. (2010). Physical exercise and reduction of pain in adults with lower limb osteoarthritis: a systematic review. Journal of Back and Musculoskeletal Rehabilitation, 23(4), 175-186.

16. Esch, T., Duckstein, J., Welke, J., Stefano, G. y Braun, V. (2007). Mind/ body techniques for physiological and psychological stress reduction: stress management via Taiji training - a pilot study. Medical Science Monitor, 13(11), 488-497.

17. Faber, M.J., Bosscher, R.J., Chin, A., Paw, M.J. y Van Wieringen, P.C. (2006). Effects of exercise programs on falls and mobility in frail and pre-frail older adults: A multicenter randomized controlled trial. Archives of Physical Medicine y Rehabilitation, 87(7), 885-896.

18. Fransen, M., Nairn, L., Winstanley, J., Lam, P. y Edmonds, J. (2007). Physical activity for osteoarthritis management: a randomized controlled clinical trial evaluating hydrotherapy or tai chi classes. Arthritis $y$ Rheumatism, 57(3), 407-414. 


\begin{tabular}{|c|c|c|c|c|c|c|}
\hline Autor & Titulo & Muestra & Edades & Intervención & Variables & Conclusiones \\
\hline $\begin{array}{l}\text { Liu, Yao } \\
(2010)\end{array}$ & $\begin{array}{l}\text { Influence of } 8 \text {-week sha- } \\
\text { dow boxing exercise on } \\
\text { the indexes for evaluating } \\
\text { sleep behavior in elderly } \\
\text { people. }\end{array}$ & 82 & 66 & $\begin{array}{l}8 \text { semanas } \\
5 \text { por semana } \\
30 \text { minutos }\end{array}$ & PSQI & $\begin{array}{l}\text { Eight-week shadowboxing exercise can signi- } \\
\text { ficantly improve the indexes of sleep behavior } \\
\text { in older people. }\end{array}$ \\
\hline $\begin{array}{l}\text { Nguyen } \\
\text { y Kruse } \\
(2012)\end{array}$ & $\begin{array}{l}\text { A randomized controlled } \\
\text { trial of Tai chi for ba- } \\
\text { lance, sleep quality and } \\
\text { cognitive performance in } \\
\text { elderly Vietnamese. }\end{array}$ & 96 & 69 & $\begin{array}{l}26 \text { semanas } \\
2 \text { por semana } \\
45 \text { minutos }\end{array}$ & PSQI & $\begin{array}{l}\text { Tai chi exercise may have a positive effect } \\
\text { on sleep quality, balance, and cognitive per- } \\
\text { formance of the community-dwelling older } \\
\text { people in Vietnam }\end{array}$ \\
\hline $\begin{array}{l}\text { Esch, Duck- } \\
\text { stein, Welke, } \\
\text { Stefano } \\
\text { y Braun } \\
(2007)\end{array}$ & $\begin{array}{l}\text { Mind/body techniques } \\
\text { for physiological and } \\
\text { psychological stress re- } \\
\text { duction: stress manage- } \\
\text { ment via Taiji training - a } \\
\text { pilot study. }\end{array}$ & 9 & & 18 semanas & $\begin{array}{l}\text { Cortisol, } \\
\text { SF36, } \\
\text { PSS14... }\end{array}$ & $\begin{array}{l}\text { Subjective health increased, stress decreased } \\
\text { (objectively and subjectively) during TC prac- } \\
\text { tice. }\end{array}$ \\
\hline $\begin{array}{l}\text { Faber, M.J., } \\
\text { Bosscher, } \\
\text { R.J., Chin, } \\
\text { A., Paw, M.J. } \\
\text { y Van Wie- } \\
\text { ringen, P.C. } \\
(2006) \text {. }\end{array}$ & $\begin{array}{l}\text { Effects of exercise pro- } \\
\text { grams on falls and mobi- } \\
\text { lity in frail and pre-frail } \\
\text { older adults: A multicen- } \\
\text { ter randomized contro- } \\
\text { lled trial. }\end{array}$ & 278 & $85+-6$ & $\begin{array}{l}20 \text { semanas } \\
52 \text { sem de } \\
\text { seguimiento }\end{array}$ & $\begin{array}{l}\text { Caídas, } \\
\text { escalas } \\
\text { físicas, } \\
\text { reportes de } \\
\text { inciden- } \\
\text { cias }\end{array}$ & $\begin{array}{l}\text { Fall-preventive moderate intensity group-exer- } \\
\text { cise programs have positive effects on falling } \\
\text { and physical performance in pre-frail, but not } \\
\text { in frail elderly. }\end{array}$ \\
\hline $\begin{array}{l}\text { Fransen, M., } \\
\text { Nairn, L., } \\
\text { Winstanley, } \\
\text { J., Lam, P. y } \\
\text { Edmonds, J. } \\
(2007) . \\
\end{array}$ & $\begin{array}{l}\text { Physical activity for os- } \\
\text { teoarthritis management: } \\
\text { a randomized controlled } \\
\text { clinical trial evaluating } \\
\text { hydrotherapy or tai chi } \\
\text { classes. }\end{array}$ & $\begin{array}{l}152 \\
56 \text { en tai } \\
\text { chi }\end{array}$ & +60 & 12 semanas & $\begin{array}{l}\text { Dolor y } \\
\text { función fí- } \\
\text { sica GHQ } \\
12 \ldots\end{array}$ & $\begin{array}{l}\text { Access to either hydrotherapy or Tai Chi classes } \\
\text { can provide large and sustained improvements } \\
\text { in physical function for many older, sedentary } \\
\text { individuals with chronic hip or knee OA. }\end{array}$ \\
\hline Gao (2013) & $\begin{array}{l}\text { Effect of } 3 \text {-month tai } \\
\text { chi exercise on heart and } \\
\text { lung capability of elder } \\
\text { people. Proceedings of } \\
\text { the } 2013 \text {. }\end{array}$ & 50 & $\begin{array}{l}\text { 61-71 } \\
\text { ańos }\end{array}$ & $\begin{array}{l}12 \text { semanas } \\
5 \text { por semana } \\
55-60 \text { min }\end{array}$ & $\begin{array}{c}\text { Presión } \\
\text { sanguínea }\end{array}$ & \\
\hline $\begin{array}{l}\text { Gatts, S. y } \\
\text { Woollaco- } \\
\text { tt, M.H. } \\
(2006)\end{array}$ & $\begin{array}{l}\text { Neural mechanisms un- } \\
\text { derlying balance impro- } \\
\text { vement with short term } \\
\text { Tai Chi training. }\end{array}$ & 22 & $68-92$ & $\begin{array}{l}4 \text { semanas } \\
5 \text { por semana } \\
90 \text { minutos }\end{array}$ & $\begin{array}{c}\text { Electro- } \\
\text { miograma }\end{array}$ & $\begin{array}{l}\text { Enhanced neuromuscular responses contro- } \\
\text { lling the ankle joint of the perturbed leg. Fast, } \\
\text { accurate neuromuscular activation is crucial } \\
\text { for efficacious response to slips or trips }\end{array}$ \\
\hline $\begin{array}{l}\text { Gemmell, C. } \\
\text { y Leathem, } \\
\text { J.M. (2006). }\end{array}$ & $\begin{array}{l}\text { A study investigating the } \\
\text { effects of Tai Chi Chuan: } \\
\text { Individuals with trauma- } \\
\text { tic brain injury compared } \\
\text { to controls. }\end{array}$ & 18 & & 3 semanas & $\begin{array}{l}\text { SF36 y } \\
\text { RSES }\end{array}$ & $\begin{array}{l}\text { Tai Chi provides short-term benefits after TBI, } \\
\text { with rigorous outcome measurement needed } \\
\text { to examine long-term benefits. }\end{array}$ \\
\hline $\begin{array}{l}\text { Gong } \\
(2009)\end{array}$ & $\begin{array}{l}\text { Taiji chuan effect on } \\
\text { community indicators } \\
\text { of blood lipid in middle } \\
\text { aged and old women. }\end{array}$ & 80 & $60-80$ & $\begin{array}{l}20 \text { semanas } \\
1 \text { por semana } \\
60 \text { minutos }\end{array}$ & & \\
\hline $\begin{array}{l}\text { Ham- } \\
\text { mond, A. y } \\
\text { Freeman, K. } \\
\text { (2006). }\end{array}$ & $\begin{array}{l}\text { Community patient edu- } \\
\text { cation and exercise for } \\
\text { people with fibromyalgia: } \\
\text { A parallel group rando- } \\
\text { mized controlled trial. }\end{array}$ & 97 & 48,5 & 12 semanas & $\begin{array}{c}\text { FIQ, artri- } \\
\text { tis... }\end{array}$ & $\begin{array}{l}\text { Short-term improvements resulted from the } \\
\text { education -exercise programme but were not } \\
\text { sustained. Appropriate selection may improve } \\
\text { efficacy }\end{array}$ \\
\hline
\end{tabular}




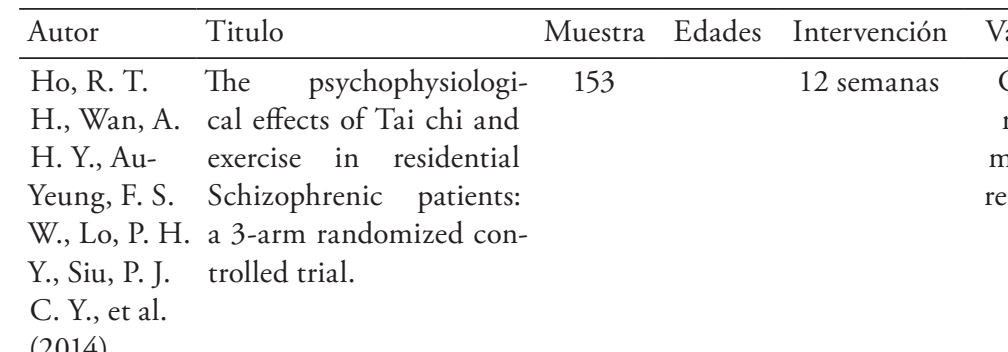

$$
\text { (2014) }
$$

\begin{tabular}{|c|c|c|c|c|}
\hline Hui (2009) & $\begin{array}{l}\text { Evaluation of energy ex- } \\
\text { penditure and cardiovascu- } \\
\text { lar health effects from tai } \\
\text { chi and walking exercise. }\end{array}$ & 374 & $\begin{array}{l}12 \text { semanas } \\
5 \text { por semana } \\
60 \text { minutos }\end{array}$ & $\begin{array}{l}\text { Nivel de } \\
\text { lípidos }\end{array}$ \\
\hline
\end{tabular}

\begin{tabular}{|c|c|c|c|c|c|c|}
\hline $\begin{array}{l}\text { Irwin, M.R., } \\
\text { Olmstead, } \\
\text { R. y Oxman, }\end{array}$ & $\begin{array}{l}\text { Augmenting immune res- } \\
\text { ponses to varicella zoster } \\
\text { virus in older adults: A }\end{array}$ & 112 & $59-86$ & 25 semanas & SF36 & $\begin{array}{l}\text { Tai Chi augments resting levels of VZV-speci- } \\
\text { fic CMI and boosts VZV-CMI of the varicella } \\
\text { vaccine. }\end{array}$ \\
\hline
\end{tabular}

(2007) trial of Tai Chi.

\begin{tabular}{|c|c|c|c|c|c|}
\hline $\begin{array}{l}\text { Jahnke, } \\
\text { R.A., Lar- } \\
\text { key, L.K. y } \\
\text { Rogers, C. }\end{array}$ & $\begin{array}{l}\text { Dissemination and bene- } \\
\text { fits of a replicable Tai Chi } \\
\text { and Qigong program for } \\
\text { older adults. }\end{array}$ & 330 & 73 & $\begin{array}{l}\text { Nivel de } \\
\text { estrés, } \\
\text { sueño }\end{array}$ & $\begin{array}{l}\text { A train-the-facilitator model for Tai Chi Easy } \\
\text { is easily disseminated to older adults and may } \\
\text { promote a sustainable alternative exercise, yiel- } \\
\text { ding favorable quality of life benefits. }\end{array}$ \\
\hline
\end{tabular}
(2010).

\begin{tabular}{|c|c|c|c|c|c|c|}
\hline $\begin{array}{l}\text { Johans- } \\
\text { son, M., } \\
\text { Hassmén, P. } \\
\text { y Jouper, J. } \\
\text { (2008). }\end{array}$ & $\begin{array}{l}\text { Acute effects of Qigong } \\
\text { exercise on mood and } \\
\text { anxiety. }\end{array}$ & 41 & $\begin{array}{l}\text { Todas } \\
\text { edades }\end{array}$ & $\begin{array}{l}12 \text { semanas } \\
3 \text { por semana } \\
60 \text { minutos }\end{array}$ & $\begin{array}{l}\text { Compara- } \\
\text { ción entre } \\
30 \text { min y } \\
60 \text { min. }\end{array}$ & $\begin{array}{l}\text { This finding is important for those having little } \\
\text { time or motivation to engage in activities of lon- } \\
\text { ger durations. In addition, health professionals } \\
\text { prescribing exercise for health benefits can pres- } \\
\text { cribe shorter exercise sessions with confidence } \\
\text { knowing that positive psychological effects can } \\
\text { also occur after a shorter exercise bout. }\end{array}$ \\
\hline
\end{tabular}

\begin{tabular}{|c|c|c|c|c|c|}
\hline $\begin{array}{l}\text { Logghe } \\
\text { (2009) }\end{array}$ & $\begin{array}{l}\text { Lack of effect of tai chi } \\
\text { chuan in preventing falls } \\
\text { in elderly people living at }\end{array}$ & 269 & 77 & $\begin{array}{c}12 \text { semanas } \\
2 \text { por semana } \\
60 \text { minutos }\end{array}$ & $\begin{array}{c}\text { Presión } \\
\text { sanguínea }\end{array}$ \\
\hline
\end{tabular}
home: a randomized clinical trial.

\begin{tabular}{|c|c|c|c|c|c|c|}
\hline $\begin{array}{l}\text { Li, F., } \\
\text { Fisher, K.J., } \\
\text { Harmer, P. y } \\
\text { McAuley, E. } \\
\text { (2005). }\end{array}$ & $\begin{array}{l}\text { Falls self-efficacy as a me- } \\
\text { diator of fear of falling in } \\
\text { an exercise intervention } \\
\text { for older adults }\end{array}$ & 256 & $\begin{array}{l}77+- \\
4.95\end{array}$ & $\begin{array}{c}24 \text { semanas } \\
3 \text { por semana } \\
60 \text { minutos }\end{array}$ & $\begin{array}{l}\text { Riesgo de } \\
\text { caída }\end{array}$ & $\begin{array}{l}\text { Tai Chi participants, who evidenced impro- } \\
\text { vement in falls self-efficacy over the course of } \\
\text { the intervention, reported greater reductions } \\
\text { in fear of falling, compared with those in the } \\
\text { stretching control condition. Results suggest } \\
\text { that exercise interventions designed to impro- } \\
\text { ve falls self-efficacy are likely to reduce fear of } \\
\text { falling in older adults }\end{array}$ \\
\hline $\begin{array}{l}\text { Li, F., } \\
\text { Harmer, P., } \\
\text { Fisher, K.J., } \\
\text { McAuley, E., } \\
\text { Chaumeton, } \\
\text { N., Eck- }\end{array}$ & $\begin{array}{l}\text { Tai Chi and fall reduc- } \\
\text { tions in older adults: a } \\
\text { randomized controlled } \\
\text { trial. }\end{array}$ & 256 & 44.48 & $\begin{array}{c}24 \text { semanas } \\
3 \text { por semana } \\
60 \text { minutos }\end{array}$ & $\begin{array}{l}\text { Caídas, } \\
\text { heridas, } \\
\text { compara- } \\
\text { tiva con } \\
\text { estira- } \\
\text { mientos }\end{array}$ & $\begin{array}{l}\text { A three-times-per-week, 6-month Tai Chi pro- } \\
\text { gram is effective in decreasing the number of } \\
\text { falls, the risk for falling, and the fear of falling, } \\
\text { and it improves functional balance and physi- } \\
\text { cal performance in physically inactive persons } \\
\text { aged } 70 \text { years or older. }\end{array}$ \\
\hline
\end{tabular}
strom, E. et al. (2005)

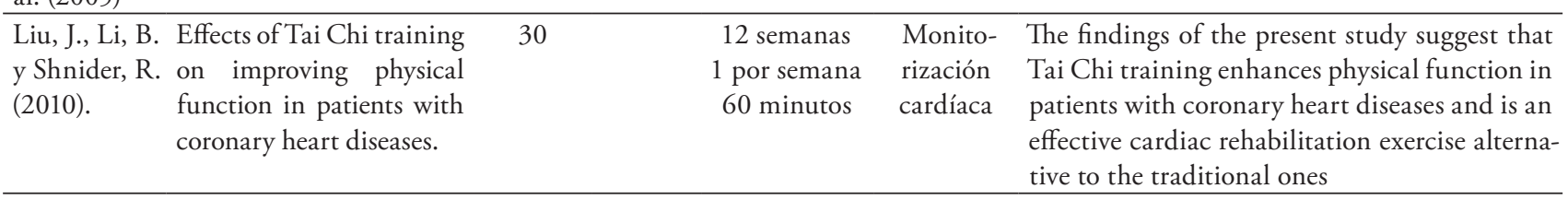




\begin{tabular}{|c|c|c|c|c|c|c|}
\hline Autor & Titulo & Muestra & Edades & Intervención & Variables & Conclusiones \\
\hline $\begin{array}{l}\text { Liu, X., } \\
\text { Miller y.D., } \\
\text { Burton, } \\
\text { N.W. y } \\
\text { Brown, W.J. } \\
\text { (2010) }\end{array}$ & $\begin{array}{l}\text { Preliminary study of the } \\
\text { effects of Tai Chi and } \\
\text { Qigong medical exercise } \\
\text { on indicators of meta- } \\
\text { bolic syndrome, glycae- }\end{array}$ & 11 & $42-65$ & $\begin{array}{c}12 \text { semanas } \\
3 \text { por semana } \\
75 \text { minutos }\end{array}$ & $\begin{array}{c}\text { BMI, } \\
\text { colesterol, } \\
\text { glucose, } \\
\text { HBAlc... }\end{array}$ & $\begin{array}{l}\text { The programme was feasible and acceptable } \\
\text { and participants showed improvements in me- } \\
\text { tabolic and psychological variables. A larger } \\
\text { controlled trial is now needed to confirm these } \\
\text { promising preliminary results. }\end{array}$ \\
\hline
\end{tabular}

(2010). mia control, health-re-

lated quality of life, and

psychological health

in adults with elevated

blood glucose.

\begin{tabular}{llrrr}
\hline Maciaszek, & Effect of tai chi on body 49 & $60-82$ & 18 semanas & Equilibrio Thus, an 18-week period of Tai Chi exercises \\
J., Osiski, & balance: Randomized & 2 por semana & twice a week for 45 min is beneficial for dyna- \\
W., Sze- & controlled trial in men & 45 minutos & mic balance. It can be important for reducing \\
klicki, R. y & with osteopenia or osteo- & & fall risk factors
\end{tabular}

Stemplewski, porosis.

R. (2007)

\begin{tabular}{|c|c|c|c|c|c|c|}
\hline $\begin{array}{l}\text { McGibbon, } \\
\text { C.A., Krebs, } \\
\text { D.E., Parker, } \\
\text { S.W., Scar- } \\
\text { borough, } \\
\text { D.M., } \\
\text { Wayne, P.M. }\end{array}$ & $\begin{array}{l}\text { Tai Chi and vestibular } \\
\text { rehabilitation improve } \\
\text { vestibulopathic gait via } \\
\text { different neuromuscular } \\
\text { mechanisms: Preliminary } \\
\text { report. }\end{array}$ & 36 & +60 & 10 semanas & $\begin{array}{c}\text { Medidas } \\
\text { vestibu- } \\
\text { lares de } \\
\text { equilibrio }\end{array}$ & $\begin{array}{l}\text { The TC group's improvements were associated } \\
\text { with reorganized lower extremity neuromus- } \\
\text { cular patterns, which appear to promote a } \\
\text { faster gait and reduced excessive hip compen- } \\
\text { sation. These data support a growing body of } \\
\text { evidence that Tai Chi may be a valuable com- } \\
\text { plementary treatment for vestibular disorders. }\end{array}$ \\
\hline
\end{tabular}

y Wolf, S.L.

(2005)

\begin{tabular}{|c|c|c|c|c|c|c|}
\hline $\begin{array}{l}\text { Motivala, } \\
\text { S., Sollers, } \\
\text { J., Thayer, J. } \\
\text { y Irwin, M. } \\
(2006) \text {. }\end{array}$ & $\begin{array}{l}\text { Tai chi chuan acutely de- } \\
\text { creases sympathetic ner- } \\
\text { vous system activity in } \\
\text { older adults. }\end{array}$ & 32 & +60 & $\begin{array}{l}25 \text { semanas } \\
1 \text { por semana } \\
20 \text { minutos }\end{array}$ & $\begin{array}{l}\text { Actividad } \\
\text { simpática }\end{array}$ & $\begin{array}{l}\text { TCC performance led to acute decreases in } \\
\text { sympathetic activity, which could not be ex- } \\
\text { plained by physical activity alone. Further stu- } \\
\text { dy is needed to determine whether the acute } \\
\text { salutary effects of TCC on autonomic functio- } \\
\text { ning are sustained with ongoing practice in } \\
\text { older adults. }\end{array}$ \\
\hline $\begin{array}{l}\text { Mustian, } \\
\text { K.M., } \\
\text { Palesh, O. y } \\
\text { Flecksteiner, } \\
\text { S. (2008). }\end{array}$ & $\begin{array}{l}\text { Tai Chi Chuan for breast } \\
\text { cancer survivors. }\end{array}$ & 21 & $52+-9$ & $\begin{array}{l}12 \text { semanas } \\
3 \text { por semana } \\
60 \text { minutos }\end{array}$ & $\begin{array}{l}\text { BMI, } \\
\text { capacidad } \\
\text { aeróbica, } \\
\text { flexibi- } \\
\text { lidad, } \\
\text { calidad de } \\
\text { vida... }\end{array}$ & $\begin{array}{l}\text { The TCC group exhibited significant improve- } \\
\text { ments in functional capacity and QOL. These } \\
\text { data suggest that TCC may enhance functio- } \\
\text { nal capacity and QOL among breast cancer } \\
\text { survivors. }\end{array}$ \\
\hline $\begin{array}{l}\text { Peppone, } \\
\text { L.J., Mus- } \\
\text { tian, K.M., } \\
\text { Janelsins, } \\
\text { M.C., Pa- } \\
\text { lesh, O.G., } \\
\text { Rosier, R.N. } \\
\text { et al. (2010). }\end{array}$ & $\begin{array}{l}\text { Effects of a structured } \\
\text { weight-bearing exercise } \\
\text { program on bone meta- } \\
\text { bolism among breast can- } \\
\text { cer survivors: A feasibility } \\
\text { trial. }\end{array}$ & 16 & 53 & $\begin{array}{l}12 \text { semanas } \\
3 \text { por semana } \\
60 \text { minutos }\end{array}$ & $\begin{array}{l}\text { Densi- } \\
\text { tometría } \\
\text { ósea }\end{array}$ & $\begin{array}{l}\text { This pilot study suggests that weight-bearing } \\
\text { exercise exerts positive effects on BL, through } \\
\text { increased bone formation and decreased bone } \\
\text { resorption. Further examinations of the in- } \\
\text { fluence of TCC on bone health are warranted. }\end{array}$ \\
\hline $\begin{array}{l}\text { Shen, C., } \\
\text { Williams, } \\
\text { J.S., Chyu, } \\
\text { M.P., Ste- } \\
\text { phens, A., } \\
\text { Chauncey, } \\
\text { K.B. et al. } \\
\text { (2007) }\end{array}$ & $\begin{array}{l}\text { Comparison of the effects } \\
\text { of Tai Chi and resistance } \\
\text { training on bone meta- } \\
\text { bolism in the elderly: A } \\
\text { feasibility study. }\end{array}$ & 28 & $78+-1.3$ & $\begin{array}{l}24 \text { semanas } \\
3 \text { por semana } \\
40 \text { minutos }\end{array}$ & $\begin{array}{l}\text { Fosfatasa } \\
\text { alcalina, } \\
\text { pyridino- } \\
\text { line, PTH } \\
\text { y calico y } \\
\text { calico en } \\
\text { orina }\end{array}$ & $\begin{array}{l}\text { These findings support that TC is beneficial } \\
\text { for increasing bone formation in elderly, and } \\
\text { long-term application is needed to substantiate } \\
\text { the effect of TC as an alternative exercise in } \\
\text { promotion of bone health. }\end{array}$ \\
\hline
\end{tabular}




\begin{tabular}{|c|c|c|c|c|c|c|}
\hline Autor & Titulo & Muestra & Edades & Intervención & Variables & Conclusiones \\
\hline $\begin{array}{l}\text { Song, R., } \\
\text { Lee, E., } \\
\text { Lam, P. y } \\
\text { Bae, S. } \\
(2010) .\end{array}$ & $\begin{array}{l}\text { Effects of tai chi exercise } \\
\text { on pain, balance, muscle } \\
\text { strength, and perceived } \\
\text { difficulties in physical } \\
\text { functioning in older wo- } \\
\text { men with osteoarthritis: } \\
\text { A randomized clinical } \\
\text { trial. }\end{array}$ & 72 & +60 & $\begin{array}{l}12 \text { semanas } \\
1 \text { por semana } \\
60 \text { minutos }\end{array}$ & $\begin{array}{l}\text { Dolor, } \\
\text { marca- } \\
\text { dores rehu- } \\
\text { matoides }\end{array}$ & $\begin{array}{l}\text { Older women with OA were able to safely per- } \\
\text { form the } 12 \text { forms of Sun-style tai chi exercise } \\
\text { for } 12 \text { weeks, and this was effective in impro- } \\
\text { ving their arthritic symptoms, balance, and } \\
\text { physical functioning. A longitudinal study } \\
\text { with a larger sample size is now needed to } \\
\text { confirm the potential use of tai chi exercise in } \\
\text { arthritis management. }\end{array}$ \\
\hline $\begin{array}{l}\text { Soto, J.R. } \\
(2006) \text {. }\end{array}$ & $\begin{array}{l}\text { Influencia de la práctica } \\
\text { del Taiji Chuan en la con- } \\
\text { dición física, funcional y } \\
\text { saludable de las personas } \\
\text { mayores. }\end{array}$ & 66 & 69.73 & $\begin{array}{l}12 \text { semanas } \\
3 \text { por semana } \\
60 \text { minutos }\end{array}$ & $\begin{array}{c}\text { Presión } \\
\text { sanguí- } \\
\text { nea, frec } \\
\text { cardiaca, } \\
\text { equilibrio y } \\
\text { flexibilidad } \\
\end{array}$ & $\begin{array}{l}\text { El Tai Chi-Chuan por lo tanto, puede ser una } \\
\text { actividad física adecuada para mejorar la con- } \\
\text { dición física funcional y saludable de las perso- } \\
\text { nas mayores }\end{array}$ \\
\hline $\begin{array}{l}\text { Stenlund, T., } \\
\text { Birgander, } \\
\text { L., Lindahl, } \\
\text { B., Nils- }\end{array}$ & $\begin{array}{l}\text { Effects of Qigong in pa- } \\
\text { tients with burnout: a } \\
\text { randomized controlled } \\
\text { trial. }\end{array}$ & 82 & $\begin{array}{c}44.3+- \\
9.1\end{array}$ & $\begin{array}{l}12 \text { semanas } \\
2 \text { por semana } \\
60 \text { minutos }\end{array}$ & $\begin{array}{l}\text { Burnout, } \\
\text { fatiga, } \\
\text { ansiedad y } \\
\text { depresión }\end{array}$ & $\begin{array}{l}\text { In this study, a Qigong intervention twice a } \\
\text { week for } 12 \text { weeks had no additional effect be- } \\
\text { yond basic care for patients with burnout }\end{array}$ \\
\hline
\end{tabular}

son, L. y

Ahlgren, C. (2009)

\begin{tabular}{|c|c|c|c|c|c|c|}
\hline $\begin{array}{l}\text { Taylor- } \\
\text { Piliae, R.E., } \\
\text { Newell, } \\
\text { K.A., Che- } \\
\text { rin, R, Lee, } \\
\text { M.J., King, } \\
\text { A.C. et al. } \\
\text { (2010) }\end{array}$ & $\begin{array}{l}\text { Effects of Tai Chi and } \\
\text { Western Exercise On } \\
\text { Physical and Cognitive } \\
\text { Functioning In Healthy } \\
\text { Community-Dwelling } \\
\text { Older Adults. }\end{array}$ & 76 & $69+-5.8$ & $\begin{array}{l}12 \text { semanas } \\
5 \text { por semana } \\
60 \text { minutos }\end{array}$ & $\begin{array}{l}\text { Función } \\
\text { física, } \\
\text { equilibrio, } \\
\text { Fortaleza, } \\
\text { flexibi- } \\
\text { lidad, } \\
\text { Resistencia } \\
\text { cardíaca }\end{array}$ & $\begin{array}{l}\text { The TC and WE interventions resulted in di- } \\
\text { fferential improvements in physical functio- } \\
\text { ning among generally healthy older adults. TC } \\
\text { led to improvement in an indicator of cogniti- } \\
\text { ve functioning that was maintained through } \\
12 \text { weeks }\end{array}$ \\
\hline Sun (2010) & $\begin{array}{l}\text { Research on the effect of } \\
\text { middle-aging and elderly } \\
\text { high blood pressure pa- } \\
\text { tients quality of life by } \\
\text { practice Tai chi. }\end{array}$ & 88 & $40-70$ & $\begin{array}{l}12 \text { semanas } \\
\text { seguimientos } \\
6 \text { meses }\end{array}$ & $\begin{array}{l}\text { SF36, } \\
\text { presión } \\
\text { sanguínea }\end{array}$ & \\
\hline $\begin{array}{l}\text { Tsang, H., } \\
\text { Fung, K., } \\
\text { Chan, A., } \\
\text { Lee, G. y } \\
\text { Chan, F. } \\
(2006) .\end{array}$ & $\begin{array}{l}\text { Effect of a qigong exerci- } \\
\text { se programme on elderly } \\
\text { with depression. }\end{array}$ & 82 & & $\begin{array}{l}16 \text { semanas } \\
5 \text { por semana } \\
60 \text { minutos }\end{array}$ & $\begin{array}{l}\text { Humor, } \\
\text { autoe- } \\
\text { ficacia, } \\
\text { bienestar, } \\
\text { autocon- } \\
\text { cepto... }\end{array}$ & $\begin{array}{l}\text { This report shows that regular qigong practice } \\
\text { could relieve depression, improve self-efficacy } \\
\text { and personal well being among elderly persons } \\
\text { with chronic physical illness and depression. }\end{array}$ \\
\hline $\begin{array}{l}\text { Tsang, W. y } \\
\text { Hui-Chan, } \\
\text { C. }(2005) \text {. }\end{array}$ & $\begin{array}{l}\text { Comparison of muscle } \\
\text { torque, balance, and con- } \\
\text { fidence in older tai chi } \\
\text { and healthy adults. }\end{array}$ & 24 & $\begin{array}{c}69.3+- \\
5.0\end{array}$ & $\begin{array}{l}\text { Practicantes } \\
\text { habituales } \\
\text { frente a otros } \\
\text { que no }\end{array}$ & $\begin{array}{l}\text { Test iso- } \\
\text { cinéticos } \\
\text { concén- } \\
\text { tricos y } \\
\text { excéntri- } \\
\text { cos }\end{array}$ & $\begin{array}{l}\text { Long-term Tai Chi practitioners had better } \\
\text { knee muscle strength, less body sway in per- } \\
\text { turbed single-leg stance, and greater balance } \\
\text { confidence }\end{array}$ \\
\hline $\begin{array}{l}\text { Voukelatos, } \\
\text { A., Cum- } \\
\text { ming, R., }\end{array}$ & $\begin{array}{l}\text { A randomized, controlled } \\
\text { trial of Tai Chi for the } \\
\text { prevention of falls: the }\end{array}$ & 702 & & $\begin{array}{l}24 \text { semanas } \\
1 \text { por semana } \\
60 \text { minutos }\end{array}$ & $\begin{array}{l}\text { Caídas y } \\
\text { equilibrio }\end{array}$ & $\begin{array}{l}\text { Participation in once per week tai chi classes } \\
\text { for } 16 \text { weeks can prevent falls in relatively } \\
\text { healthy community-dwelling older people. }\end{array}$ \\
\hline
\end{tabular}

Lord, S.R. central Sydney tai chi

y Rissel, C. trial. 


\begin{tabular}{|c|c|c|c|c|c|c|}
\hline Autor & Titulo & Muestra & Edades & Intervención & Variables & Conclusiones \\
\hline $\begin{array}{l}\text { Wang, C., } \\
\text { Roubenoff, } \\
\text { R., Lau, J., } \\
\text { Kalish, R., } \\
\text { Schmid, } \\
\text { C.H. et al. }\end{array}$ & $\begin{array}{l}\text { Effect of tai chi in adults } \\
\text { with rheumatoid arthritis. }\end{array}$ & 10 & $48+-10$ & 12 semanas & $\begin{array}{c}\text { SF36, } \\
\text { inflama- } \\
\text { ción, dolor, } \\
\text { vitalidad... }\end{array}$ & $\begin{array}{l}\text { The results demonstrate that Tai Chi seems } \\
\text { to be associated with trends to improvement } \\
\text { in disease activity that relates to both symp- } \\
\text { toms of pain and the cognitive coping pro- } \\
\text { cess, which in turn is related to physical and } \\
\text { psychological disability }\end{array}$ \\
\hline
\end{tabular}

(2005)

\begin{tabular}{|c|c|c|c|c|c|c|}
\hline $\begin{array}{l}\text { Wang, J. } \\
(2008)\end{array}$ & $\begin{array}{l}\text { Effects of Tai Chi exerci- } \\
\text { se on patients with type } 2 \\
\text { diabetes. }\end{array}$ & 12 & $\begin{array}{c}66.5+- \\
8.5\end{array}$ & 8 semanas & $\begin{array}{l}\text { Glucosa, } \\
\text { insulina, } \\
\text { peptido C }\end{array}$ & $\begin{array}{l}\text { The } 8 \text {-week Tai Chi intervention therefore } \\
\text { showed benefits on health status of patients } \\
\text { with type } 2 \text { diabetes. }\end{array}$ \\
\hline $\begin{array}{l}\text { Wolf, S., } \\
\text { O'Grady, } \\
\text { M., Easley, } \\
\text { K., Guo y., } \\
\text { Kressig, R. } \\
\text { et al. (2006). }\end{array}$ & $\begin{array}{l}\text { The influence of intense } \\
\text { Tai Chi training on phy- } \\
\text { sical performance and he- } \\
\text { modynamic outcomes in } \\
\text { transitionally frail, older } \\
\text { adults }\end{array}$ & 311 & +70 & 48 semanas & $\begin{array}{l}\text { BMI, } \\
\text { Tensión } \\
\text { Arterial, } \\
\text { pulsacio- } \\
\text { nes }\end{array}$ & $\begin{array}{l}\text { TC significantly improved chair-rise and car- } \\
\text { diovascular performance. Because TC training } \\
\text { reduced fall occurrences in this cohort, factors } \\
\text { influencing functional and cardiovascular } \\
\text { improvements may also favorably impact fall } \\
\text { events. }\end{array}$ \\
\hline $\begin{array}{l}\text { Woo, J., } \\
\text { Hong, A., } \\
\text { Lau, E. y } \\
\text { Lynn, H. } \\
(2007) .\end{array}$ & $\begin{array}{l}\text { A randomized controlled } \\
\text { trial of tai chi and resis- } \\
\text { tance exercise on bone } \\
\text { health, muscle strength } \\
\text { and balance in commu- } \\
\text { nity-living elderly people. }\end{array}$ & 180 & $65-74$ & $\begin{array}{l}52 \text { semanas } \\
3 \text { por semana } \\
60 \text { minutos }\end{array}$ & $\begin{array}{c}\text { Fumador y } \\
\text { flexibili- } \\
\text { dad, For- } \\
\text { taleza en } \\
\text { cuádriceps }\end{array}$ & $\begin{array}{l}\text { The beneficial effects of TC or RTE on mus- } \\
\text { culoskeletal health are modest and may not } \\
\text { translate into better clinical outcomes }\end{array}$ \\
\hline $\begin{array}{l}\text { Yang, Y., } \\
\text { Verkuilen, } \\
\text { J., Rosegren, } \\
\text { K., Mariani, } \\
\text { R., Grubi- } \\
\text { sich, S. et al. } \\
\text { (2008). }\end{array}$ & $\begin{array}{l}\text { The beneficial effects of } \\
\text { Tai Chi Chuan on blood } \\
\text { pressure and lipid profile } \\
\text { and anxiety status in a } \\
\text { randomized controlled } \\
\text { trial. }\end{array}$ & 152 & & $\begin{array}{c}12 \text { semanas } \\
3 \text { por semana } \\
50 \text { minutos }\end{array}$ & $\begin{array}{l}\text { Presión } \\
\text { arterial, } \\
\text { lípidos, } \\
\text { ansiedad }\end{array}$ & $\begin{array}{l}\text { Tai Chi could be used as an alternative moda- } \\
\text { lity in treating patients with mild hypertension, } \\
\text { with a promising economic effect. }\end{array}$ \\
\hline $\begin{array}{l}\text { Yeh, S.H., } \\
\text { Chuang, H., } \\
\text { Lin, L.W., } \\
\text { Hsiao, C.Y., } \\
\text { Wang, P.W. } \\
\text { et al. (2009) }\end{array}$ & $\begin{array}{l}\text { Regular Tai Chi Chuan } \\
\text { exercise improves } \mathrm{T} \text { cell } \\
\text { helper function of pa- } \\
\text { tients with type } 2 \text { dia- } \\
\text { betes mellitus with an } \\
\text { increase in T-bet trans- } \\
\text { cription factor and IL-12 } \\
\text { production. }\end{array}$ & 60 & & 12 semanasa & $\begin{array}{l}\text { Glucosa, } \\
\text { peptido C, } \\
\text { ansiedad }\end{array}$ & $\begin{array}{l}\text { A 12-week TCC exercise programme decreases } \\
\text { HbA1c levels along with an increase in the Th1 } \\
\text { reaction. A combination of TCC with medica- } \\
\text { tion may provide an even better improvement } \\
\text { in both metabolism and immunity of patients } \\
\text { with type } 2 \mathrm{DM}\end{array}$ \\
\hline $\begin{array}{l}\text { Zhang, Is- } \\
\text { hikawaTaka- } \\
\text { ta, Morita } \\
\text { y Ohta, } \\
(2006) .\end{array}$ & $\begin{array}{l}\text { The effects of Tai Chi } \\
\text { Chuan on physiological } \\
\text { function and fear of fa- } \\
\text { lling in the less robust } \\
\text { elderly: an intervention } \\
\text { study for preventing falls. }\end{array}$ & 49 & 60 & $\begin{array}{l}12 \text { semanas } \\
3 \text { por semana } \\
60 \text { minutos }\end{array}$ & $\begin{array}{l}\text { Velocidad } \\
\text { al andar, } \\
\text { equilibrio, } \\
\text { flexión }\end{array}$ & $\begin{array}{l}\text { The results suggest that a high frequency, short- } \\
\text { term TCC training program can improve ba- } \\
\text { lance, flexibility, and increase the confidence } \\
\text { of less-robust elderly. }\end{array}$ \\
\hline
\end{tabular}

Tabla 2. Meta análisis analizados en la búsqueda realizada con un total de 13 artículos.

\begin{tabular}{llll}
\hline Du, S., & Tai chi exercise for self- & Meta & Weak evidence shows that Tai chi exercise \\
Dong, J., & rated sleep quality in & análisis & has a beneficial effect in improving self-rated \\
Zhang, H., & older people: a systematic & sleep quality for older adults, suggesting that \\
Jin, S., Xu, & review and meta-analysis & Tai chi could be an effective alternative and \\
G. et al. & & complementary approach to existing therapies \\
(2015). & & for older people with sleep problems. More \\
& & rigorous experimental studies are required \\
\hline
\end{tabular}




\begin{tabular}{|c|c|c|c|c|c|}
\hline $\begin{array}{l}\text { Escalante, } \\
\text { Saavedra, } \\
\text { García-Her- } \\
\text { moso, Silva } \\
\text { y Barbosa } \\
(2010) \\
\end{array}$ & & $\begin{array}{l}\text { Revisión } \\
\text { sistemá- } \\
\text { tica }\end{array}$ & & & \\
\hline $\begin{array}{l}\text { Hall, A., } \\
\text { Maher, C., } \\
\text { Latimer, J. y } \\
\text { Ferreira, M. } \\
(2009) \\
\end{array}$ & $\begin{array}{l}\text { The Effectiveness of } \\
\text { Tai Chi for chronic } \\
\text { musculoskeletal pain } \\
\text { conditions: A systematic } \\
\text { review and meta-analysis. }\end{array}$ & $\begin{array}{l}\text { Meta } \\
\text { análisis }\end{array}$ & & & $\begin{array}{l}\text { Sus resultados arrojan } 1641 \text { y se estudia } 8 \\
\text { artículos }\end{array}$ \\
\hline $\begin{array}{l}\text { Hall A, } \\
\text { Copsey } \\
\text { B, Rich- } \\
\text { mond H, } \\
\text { Thompson J, } \\
\text { Ferreira M, } \\
\text { et al.(2017) } \\
\end{array}$ & $\begin{array}{l}\text { Effectiveness of Tai Chi } \\
\text { for Chronic Musculos- } \\
\text { keletal Pain Conditions: } \\
\text { Updated Systematic Re- } \\
\text { view and Meta-Analysis. }\end{array}$ & $\begin{array}{l}\text { Meta } \\
\text { análisis } \\
\text { actuali- } \\
\text { zado del } \\
\text { anterior }\end{array}$ & & $\begin{array}{l}15 \text { estudios } \\
\text { con os- } \\
\text { teoartritis, } \\
\text { dolor de } \\
\text { espalda y } \\
\text { de cabeza }\end{array}$ & $\begin{array}{l}\text { In order for tai chi to be recommended as } \\
\text { an effective intervention, more high-quality } \\
\text { trials with large sample sizes assessing tai chi } \\
\text { versus other evidence-based treatments at } \\
\text { short term and at long term are needed. }\end{array}$ \\
\hline $\begin{array}{l}\text { Hartley } \\
\text { L, Flowers } \\
\text { N, Lee } \\
\text { MS, Ernst } \\
\text { E, Rees K } \\
(2014) \\
\end{array}$ & $\begin{array}{l}\text { Tai chi for primary pre- } \\
\text { vention of cardiovascular } \\
\text { disease } \\
\text { (Review). }\end{array}$ & & & & \\
\hline $\begin{array}{l}\text { Jahnke, } \\
\text { R.A., Lar- } \\
\text { key, L.K., } \\
\text { Rogers, C. } \\
\text { y Lin, F. } \\
(2010) .\end{array}$ & $\begin{array}{l}\text { A comprehensive review } \\
\text { of health benefits of } \\
\text { Qigong and Tai Chi. }\end{array}$ & $\begin{array}{l}\text { Meta } \\
\text { análisis } \\
133\end{array}$ & $55-86$ & $\begin{array}{l}9 \text { estudios } \\
\text { que } \\
\text { señalan la } \\
\text { densidad } \\
\text { ósea, los } \\
\text { efectos } \\
\text { cardiopul- } \\
\text { monares, } \\
\text { función fí- } \\
\text { sica, caídas, } \\
\text { calidad de } \\
\text { vida, auto- } \\
\text { eficacia }\end{array}$ & $\begin{array}{l}\text { Research has demonstrated consistent, signifi- } \\
\text { cant results for a number of health benefits in } \\
\text { RCTs, evidencing progress toward recogni- } \\
\text { zing the similarity and equivalence of Qigong } \\
\text { and Tai Chi }\end{array}$ \\
\hline
\end{tabular}

Lee, M.S., Tai chi for rheumatoid $\quad$ Estudio

Pittler, M.H. arthritis: Systematic sistemá-

y Ernst, E. review. tico

(2007).

\begin{tabular}{|c|c|c|c|}
\hline $\begin{array}{l}\text { Lee, M.S., } \\
\text { Pittler, } \\
\text { M.H., } \\
\text { Taylor-Piliae, } \\
\text { R.E. y Ernst, } \\
\text { E. (2007). }\end{array}$ & $\begin{array}{l}\text { Tai chi for cardiovascular } \\
\text { disease and its risk fac- } \\
\text { tors: a systematic review. }\end{array}$ & $\begin{array}{l}\text { Estudio } \\
\text { sistemá- } \\
\text { tico }\end{array}$ & \\
\hline $\begin{array}{l}\text { Nery, R.M., } \\
\text { Zanini, M., } \\
\text { Ferrari, J.N., } \\
\text { Silva, C.A., } \\
\text { Farias, C. et } \\
\text { al. (2014) }\end{array}$ & $\begin{array}{l}\text { Tai Chi Chuan for } \\
\text { cardiac rehabilitation in } \\
\text { patients with coronary } \\
\text { arterial disease. }\end{array}$ & $\begin{array}{l}\text { Meta } \\
\text { análisis } \\
201 \\
\text { que se } \\
\text { quedan } \\
\text { en } 12\end{array}$ & $\begin{array}{l}\text { Preliminary evidence suggests that Tai Chi } \\
\text { Chuan can be an unconventional form of } \\
\text { cardiac rehabilitation, being an adjunctive } \\
\text { therapy in the treatment of patients with } \\
\text { stable coronary artery disease. However, the } \\
\text { methodological quality of the included arti- } \\
\text { cles and the small sample sizes clearly indicate } \\
\text { that new randomized controlled trials are } \\
\text { needed in this regard. }\end{array}$ \\
\hline
\end{tabular}


19. Gao, D.Q.(2013). Effect of 3-month tai chi exercise on heart and lung capability of elder people. Proceedings of the 2013 International Conference on Educational Research and Sports Education. Paris: Atlantis Press. 39, 86-8.

20. Gatts, S. y Woollacott, M.H. (2006). Neural mechanisms underlying balance improvement with short term Tai Chi training. Aging-Clinical y Experimental Research, 18(1), 7-19.

21. Gemmell, C. y Leathem, J.M. (2006). A study investigating the effects of Tai Chi Chuan: Individuals with traumatic brain injury compared to controls. Brain Injury, 20(2), 151-156.

22. Gong, J.C., Liu, Y.D., Zhao, D.M., Zhao, M.L. (2009).Taijishan effect on community indicators of blood lipid in middle aged and old women. Journal of Community Medicine, 7(17):34-5.

23. Hall, A., Maher, C., Latimer, J. y Ferreira, M. (2009). The Effectiveness of Tai Chi for chronic musculoskeletal pain conditions: A systematic review and meta-analysis. Arthritis and Rheumatism, 61(6), 717-724.

24. Hall A, Copsey B, Richmond H, Thompson J, Ferreira M, et al. (2017). Effectiveness of Tai Chi for Chronic Musculoskeletal Pain Conditions: Updated Systematic Review and Meta-Analysis. Physical Therapy 97(2):227-38

25. Halsted, M., Bennet, S. y Cunningham, W. (1993). Measuring coping in adolescents: An application of the ways of coping chechlist. Journal of Clinical Child Psychology, 22(3), 337-344.

26. Hammond, A. y Freeman, K. (2006). Community patient education and exercise for people with fibromyalgia: A parallel group randomized controlled trial. Clinical Rehabilitation, 20(10), 835-846.

27. Higgins, J.P.T., (2011). The Cochrane Collaboration, 2011. Cochrane Handbook for Systematic Reviews of Interventions Version 5.1.0. The Cochrane Collaboration. http://www.cochrane-handbook.org Green, S. (Eds.), (acceso 20.12.2016).

28. Ho, R. T. H., Wan, A. H. Y., Au-Yeung, F. S. W., Lo, P. H. Y., Siu, P. J. C. Y., et al. (2014). The psychophysiological effects of Tai-chi and exercise in residential Schizophrenic patients: a 3-arm randomized controlled trial. BMC Complementary and Alternative Medicine, 14, 364. http://doi.org/10.1186/1472-6882-14-364

29. Hosseini, H., Esfirizi, M.F., Marandi, S.M., and Rezaei, A. (2011). The effect of $\mathrm{Ti}$ Chi exercise on the sleep quality of the elderly residents in Isfahan, Sadeghieh elderly home. Iran Journal. Nursery Midwifery Resources. 16: 55-60.

30. Hui, S.S.C., Woo, J., Kwok, T.(2009). Evaluation of energy expenditure and cardiovascular health effects from tai chi and walking exercise. Hong Kong Medical Journal;15. S4-7.

31. Irwin, M.R., Olmstead, R. y Oxman, M.N. (2007). Augmenting immune responses to varicella zoster virus in older adults: A randomized, controlled trial of Tai Chi. Journal of the American Geriatrics Society, 55(4), 511-517.

32. Irwin, M.R., Olmstead, R. y Motivala, S.J. (2008). Improving sleep quality in older adults with moderate sleep complaints: a randomized controlled trial of Tai Chi Chih. Sleep.; 31: 1001-1008

33. Jahnke, R.A., Larkey, L.K. y Rogers, C. (2010). Dissemination and benefits of a replicable Tai Chi and Qigong program for older adults. Geriatric Nursing, 31(4), 272-280.

34. Jahnke, R.A., Larkey, L.K., Rogers, C. y Lin, F. (2010). A comprehensive review of health benefits of Qigong and Tai Chi. American Journal of Health Promotion, 24(6), e1-e25.

35. Johansson, M., Hassmén, P. y Jouper, J. (2008). Acute effects of Qigong exercise on mood and anxiety. International Journal of stress Management, 15(5), 199-207.

36. Lan, C., Chen, S.Y., Lai, J.S. y Wong, A.M. (2013). Tai Chi Chuan in medicine and health promotion. Evidence-Based Complementary and Alternative Medicine, 502131.

37. Lee, M.S., Pittler, M.H. y Ernst, E. (2007). Tai chi for rheumatoid arthritis: Systematic review. Rheumatology, 46(11), 1648-1651.
38. Lee, M.S., Pittler, M.H., Taylor-Piliae, R.E. y Ernst, E. (2007). Tai chi for cardiovascular disease and its risk factors: a systematic review. Journal of Hypertension, 25(9), 1974-1975.

39. Li, F., Fisher, K.J., Harmer, P. y McAuley, E. (2005). Falls self-efficacy as a mediator of fear of falling in an exercise intervention for older adults. Journals of Gerontology Series B: Psychological Sciences and Social Sciences, 60(1), 34-40.

40. Li, F., Harmer, P., Fisher, K.J., McAuley, E., Chaumeton, N., Eckstrom, E. et al. (2005). Tai Chi and fall reductions in older adults: a randomized controlled trial. Journals of Gerontology. Series A, Biological Sciences and Medical Sciences, 60(2), 187-194.

41. Liu, J., Li, B. y Shnider, R. (2010). Effects of Tai Chi training on improving physical function in patients with coronary heart diseases. Journal of Exercise Science y Fitness, 8(2), 78-84.

42. Liu, R. and Yao, W.Y. (2010). Influence of 8-week shadow boxing exercise on the indexes for evaluating sleep behavior in elderly people. Chinese Journal of Geriatric Care 8, 26-7.

43. Liu, X., Miller y.D., Burton, N.W. y Brown, W.J. (2010). Preliminary study of the effectss of Tai Chi and Qigong medical exercice on indicators of metabolic syndrome, glycaemic control, health-related quality of life, and psychological health in adults with elevated blood glucose. British Journal of Sports Medicine, 44(10), 704-709.

44. Logghe, I.H.J., Zeeuwe, P.E.M., Verhagen, A.P., Wijnen-Sponselee, R.M.T., Willemsen, S.P et al. (2009). Lack of effect of tai chi chuan in preventing falls in elderly people living at home: a randomised clinical trial. Journal of the American Geriatrics Society. 57, 70-5.

45. Maciaszek, J., Osiski, W., Szeklicki, R. y Stemplewski, R. (2007). Effect of tai chi on body balance: Randomized controlled trial in men with osteopenia or osteoporosis. American Journal of Chinese Medicine, 35(1), $1-9$.

46. McGibbon, C.A., Krebs, D.E., Parker, S.W., Scarborough, D.M., Wayne, P.M. y Wolf, S.L. (2005). Tai Chi and vestibular rehabilitation improve vestibulopathic gait via different neuromuscular mechanisms: Preliminary report. BMC Neurology, 5(1), 3.

47. Motivala, S., Sollers, J., Thayer, J. y Irwin, M. (2006). Tai chi chih acutely decreases sympathetic nervous system activity in older adults. Journals of Gerontology. Series A, Biological Sciences and Medical Sciences, 61(11), 1177-1180.

48. Mustata, S., Cooper, L., Langrick, N., Simon, N., Jassal, S. y Oreopoulos, D. (2005). The effect of Tai Chi exercise program on quality of life in patients on peritioneal diálisis: a pilot study. Peritoneal Dialysis International, 25(3), 291-294.

49. Mustian, K.M., Palesh, O. y Flecksteiner, S. (2008). Tai Chi Chuan for breast cancer survivors. Medicine and Sports Science, 52, 209-217.

50. Nery, R.M., Zanini, M., Ferrari, J.N., Silva, C.A., Farias, C. et al. (2014). Tai Chi Chuan for cardiac rehabilitation in patients with coronary arterial disease. Arquivos Brasileiros de Cardiologia, 102(6), 588-92.

51. Nguyen, M.H. and Kruse, A. (2012). A randomized controlled trial of Tai chi for balance, sleep quality and cognitive performance in elderly Vietnamese. Clinical Interventention. Aging. 7: 185-90

52. Peppone, L.J., Mustian, K.M., Janelsins, M.C., Palesh, O.G., Rosier R.N. et al. (2010). Effects of a structured weight-bearing exercise program on bone metabolism among breast cancer survivors: A feasibility trial. Clinical Breast Cancer, 10(3), 224-229.

53. Shen, C., Williams, J.S., Chyu, M.P., Stephens, A., Chauncey, K.B. et al. (2007). Comparison of the effects of Tai Chi and resistance training on bone metabolism in the elderly: A feasibility study. American Journal of Chinese Medicine, 35(3), 369-381.

54. Song, R., Lee, E., Lam, P. y Bae, S. (2010). Effects of tai chi exercise on pain, balance, muscle strength, and perceived difficulties in physical functioning in older women with osteoarthritis: A randomized clinical trial. Journal of Rheumatology, 30(9), 2039-2044.

55. Soto, J.R. (2006). Influencia de la práctica del Taiji Chuan en la condi- 
ción física, funcional y saludable de las personas mayores. Tesis Doctoral. Universidade da Coruña. La Coruña

56. Stenlund, T., Birgander, L., Lindahl, B., Nilsson, L. y Ahlgren, C. (2009). Effects of Qigong in patients with burnout: a randomized controlled trial. Journal of Rehabilitative Medicine, 41(9), 761-767.

57. Taylor-Piliae, R.E. y Haskell, W. (2007). Tai Chi exercise and stroke rehabilitation. Topics in Stroke Rehabilitation, 14(4), 9-22.

58. Taylor-Piliae, R.E., Newell, K.A., Cherin, R, Lee, M.J., King, A.C. et al. (2010). Effects of Tai Chi and Western Exercise On Physical and Cognitive Functioning In Healthy Community-Dwelling Older Adults. Journal of Aging and Physical Activity, 18(3), 261-279.

59. Tsang, H., Fung, K., Chan, A., Lee, G. y Chan, F. (2006). Effect of a qigong exercise programme on elderly with depression. International Journal of Geriatric Psychiatry, 21(9), 890-897.

60. Tsang, W. y Hui-Chan, C. (2005). Comparison of muscle torque, balance, and confidence in older tai chi and healthy adults. Medicine and Science in Sports and Exercise, 37(2), 280-9.

61. Voukelatos, A., Cumming, R., Lord, S.R. y Rissel, C. (2007). A randomized, controlled trial of Tai Chi for the prevention of falls: the central sydney tai chi trial. Journal of the American Geriatrics Society, 55(8), 1185-1191.

62. Wang, C., Bannuru, R., Ramel, J., Kupelnick, B., Scott, T. y Schmid, C.H. (2010). Tai Chi on psychological well-being: systematic review and meta-analysis. BMC Complementary and Alternative Medicine, 10(23).

63. Wang, C., Roubenoff, R., Lau, J., Kalish, R., Schmid, C.H. et al. (2005). Effect of tai chi in adults with rheumatoid arthritis. Rheumato$\log y, 44(5), 685-687$.

64. Wang, F., Lee, E.K., Wu, T., Benson, H., Fricchione, G. et al. (2014). The effects of tai chi on depression, anxiety, and psychological well- being: a systematic review and meta-analysis. International Journal of Behavioral Medicine, 21(4), 605-617.

65. Wang, J. (2008). Efects of Tai Chi exercice on patients with type 2 diabetes. Medicine and Sport Science, 52, 230-238.

66. Wang, W., Zhang, A., Rasmussen, B., Lin, L., Kang, S. et al. (2009). The effect of Tai Chi on psychosocial well-being: a systematic review of randomized controlled trials. Journal of Acupuncture and Meridian Studies, 2(3), 171-181.

67. Wolf, S., O'Grady, M., Easley, K., Guo y., Kressig, R. et al. (2006). The influence of intense Tai Chi training on physical performance and hemodynamic outcomes in transitionally frail, older adults. Journals of Gerontology. Series A, Biological Sciences and Medical Sciences, 61(2), 184-189.

68. Woo, J., Hong, A., Lau, E. y Lynn, H. (2007). A randomised controlled trial of tai chi and resistance exercise on bone health, muscle strength and balance in community-living elderly people. Age y Ageing, 36(3), 262-268.

69. Yang, Y., Verkuilen, J., Rosegren, K., Mariani, R., Grubisich, S. et al. (2008). The beneficial effects of Tai Chi Chuan on blood pressure and lipid profile and anxiety status in a randomized controlled trial. Journal of Alternative and Complementary Medicine (9), 747-754.

70. Yeh, S.H., Chuang, H., Lin, L.W., Hsiao, C.Y., Wang, P.W. et al. (2009) Regular Tai Chi Chuan exercise improves $\mathrm{T}$ cell helper function of patients with type 2 diabetes mellitus with an increase in T-bet transcription factor and IL-12 production. British Journal of Sports Medicine, 43(11), 845-850.

71. Zhang, J., IshikawaTakata, K. , Morita, T. y Ohta, T. (2006). The effects of Tai Chi Chuan on physiological function and fear of falling in the less robust elderly: an intervention study for preventing falls. $A r-$ chives of Gerontology and Geriatrics, 42(2), 107-116. 